\title{
ASYMPTOTIC PERTURBATION SERIES
}

\author{
BY \\ VERNON A. KRAMER( $\left.{ }^{1}\right)$
}

1. Introduction. This paper is devoted to the study of the validity in an asymptotic sense of the perturbation method as applied to an eigenvalueeigenvector problem. Conditions under which the method of identification of coefficients leads to convergent infinite series have been studied by Rellich $[1 ; 2 ; 3 ; 4]$, Nagy [1], and Wolf [1]. In more general cases, especially with unbounded operators, this method may lead to only finite or possibly nonconvergent series. We would still be interested in whether these series are valid asymptotically to the extent that they are defined. Titchmarsh [1] has investigated this problem for special differential operators. Kato $[2 ; 3]$ has derived conditions sufficient for the expansions to be valid as far as they are defined for an operator of the form $H_{0}+t H_{1}$. The corresponding results do not hold for operators with higher order terms, not even for the case $H_{0}+t^{2} H_{2}$. (See Example 3.1.) This paper gives a set of conditions sufficient for the validity of the expansions for operators defined by finite or infinite series.

Some familiarity with the formal method is assumed. For example, Chapter VI of Pauling and Wilson [1] should be sufficient.

We study operators on a Hilbert space $\mathfrak{H C}$ defined by a series (possibly finite) $H(t)=H_{0}+t H_{1}+t^{2} H_{2}+\cdots$, where $H_{0}$ is self-adjoint and the other terms are symmetric. Let $\lambda_{0}$ be an isolated eigenvalue of $H_{0}$ and $\phi_{0}$ a corresponding normalized eigenvector. We would expect $H(t)$ to have an eigenvalue converging to $\lambda_{0}$. The method of identification of coefficients leads to series $\lambda(t)=\lambda_{0}+t \lambda_{1}+t^{2} \lambda_{2}+\cdots$ and $\phi(t)=\phi_{0}+t \phi_{1}+t^{2} \phi_{2}+\cdots$ for the perturbed eigenvalue and eigenvector. We say these series are asymptotically valid to $N$ th order if there exists a perturbed eigenvalue $\lambda(t)$ and a corresponding eigenvector $\phi(t)$ such that $t^{-N}\left|\lambda(t)-\sum_{i=0}^{N} t^{i} \lambda_{i}\right|$ and $t^{-N} \| \phi(t)$ $-\sum_{i=0}^{N} t^{i} \phi_{i} \|$ tend to zero with $t$.

To guarantee the existence of the perturbed eigenvalue we impose certain semi-boundedness conditions on the operators $H_{i}$ and employ the theory of the Friedrichs extension of a semi-bounded operator as developed by Friedrichs $[1 ; 2]$ and Rellich $[5](\S 2)$.

Any direct attempt to justify the identification of coefficients as a repeated limiting process fails because the operators of our series may be unbounded.

Presented to the Society, April 28, 1956; received by the editors October 10, 1955.

( 1 ) This paper is part of the author's doctoral dissertation submitted to the University of California, Berkeley, 1954, prepared under the guidance of Professor Frantisek Wolf. The research was partially supported by the Office of Ordnance Research, U. S. Army, Contract DA-04-200-ORD-171.

The author is now at the University of California, Riverside, California. 
To avoid this difficulty we study the inverse operator. In an earlier paper (Kramer [1], hereafter referred to as $\boldsymbol{A}$ ) the author proved a variety of theorems concerning the validity of formal inverse expansions. Those theorems pertinent to the present work are listed at the end of $\$ 2$. Knowing the inverse expansion to be valid we study the formal method applied to the inverse operator with more success. Some changes in order must be made. Intuitively, when using the formal method to obtain the $n$th term of the eigenvector expansion, we first take a limit and then apply the reduced resolvent (Definition 2.1) to both sides of an equation. To justify the result we must essentially interchange these steps and apply the perturbed reduced resolvent first and then take the limit.

In Theorem 3.1 we prove the results of the identification of coefficients under our basic assumption. Using variational methods we then obtain the stronger conclusion that the eigenvalue can be expanded to twice the order of the eigenvector in Theorem 3.2. Odd order eigenvalue expansions are obtained in Theorem 3.3.

$\S \S 4$ and 5 are devoted to a study of the degenerate case. The formal proofs are restricted to first order only but splitting at higher order is discussed briefly. A point of interest in $\S 5$ is that the formal first order results are not completely verified until second order conditions are imposed.

In $\S 6$ the adiabatic transformation is used to obtain uniquely determined and "naturally" distinguished eigenvectors.

2. Preliminary lemmas. In this section we lay down certain fundamental assumptions and establish a number of lemmas for later use. The conditions on the operators of the series are the same as those used in $A$; namely, $H_{0} \geq I$, $H_{i} \geq 0$ for $i>0, t \geq 0$, and $D(H(t))$ dense for $t \leq t_{0}$. Then $H(t)$ has a Friedrichs extension $\widetilde{H}(t)$ and we study this extension instead of $H(t)$ itself. We further assume that $H_{0}$ is the Friedrichs extension of its own contraction to $D(H(t))$.

For our purpose, the most important property of the Friedrichs extension is that given by the following lemma. For a proof, see Rellich [5].

Lemma 2.1. If $A$ and $B$ are symmetric operators semi-bounded from below with $A \geq B$, and $a$ and $b$ are the least limit points of the spectrum of $\tilde{A}$ and $\widetilde{B}$ respectively, then $a \geq b$. If $\tilde{A}$ has $N$ eigenvalues less than a constart $k$, then $\widetilde{B}$ has at least $N$ eigenvalues less than $k$ and the ith eigenvalue of $\tilde{A}$ is not less than that of $\widetilde{B}$ for $i \leq N$.

Lemma 2.2. If $k$ is a fixed constant, then the number of eigenvalues of $\tilde{\boldsymbol{H}}(t)^{-1}$ above $k$ is a nonincreasing function of $t$ for $t \geq 0$.

Proof. Obviously $H\left(t_{1}\right) \leq H\left(t_{2}\right)$ for $0 \leq t_{1} \leq t_{2}$. The lemma follows immediately from the preceding lemma and the spectral mapping theorem (Stone $[1$, p. 233]).

Lemma 2.3. If $\mu_{0}$ is an isolated eigenvalue of multiplicity $L$ of $H_{0}^{-1}$ and the 
spectrum of $H_{0}^{-1}$ above $\mu_{0}$ consists of isolated eigenvalues with total multiplicity finite, then for sufficiently small $t, \tilde{H}(t)^{-1}$ has exactly $L$ eigenvalues (counting multiplicity) which converge to $\mu_{0}$ and the remainder of the spectrum of $\widetilde{H}(t)^{-1}$ is bounded away from $\mu_{0}$.

Proof. Let $E_{t}(\lambda)$ denote the resolution of the identity corresponding to $\widetilde{H}(t)^{-1}$. From Theorem $3.1 A$ we have $\widetilde{H}(t)^{-1}$ converges strongly to $H_{0}^{-1}$. Therefore, (see Stone [1, p. 390]) if $\lambda$ is not an eigenvalue of $H_{0}^{-1}$ then $\left(E_{t}(\lambda) f, g\right)$ converges to $\left(E_{0}(\lambda) f, g\right)$ for all $f, g$ in $\mathfrak{H C}$. Expanding

$$
\left\|E_{t}(\lambda) f-E_{0}(\lambda) f\right\|^{2}
$$

as an inner product we see that its limit is zero so $E_{t}(\lambda)$ converges strongly to $E_{0}(\lambda)$. Now if $J$ is any interval containing $\mu_{0}$ in its interior but no other spectral point of $H_{0}^{-1}$ and $F(t)$ is the projection of the spectral measure of $\widetilde{H}(t)^{-1}$ associated with $J$, then $F(t)$ converges strongly to $F(0)$. Consequently, the dimension of $F(t)$ cannot be less than that of $F(0)$ for sufficiently small $t$. Letting both end points of $J$ approach $\mu_{0}$ we see that $\widetilde{H}(t)^{-1}$ has at least $L$ eigenvalues or a point of the continuous spectrum which approach $\mu_{0}$. Now apply Lemma 2.2 with $k$ between $\mu_{0}$ and the next lower spectral point of $H_{0}^{-1}$. There are no points of the continuous spectrum of $\widetilde{H}(t)^{-1}$ above $k$ and the number of eigenvalues of $\widetilde{H}(t)^{-1}$ above $k$ cannot exceed that of $H_{0}^{-1}$. Therefore, there exist precisely $L$ perturbed eigenvalues which converge to $\mu_{0}$.

In the following we will use $E_{0}$ to denote the projection associated with $\mu_{0}$ and $E(t)$ for the projection associated with all of the $L$ eigenvalues of $\widetilde{H}(t)^{-1}$ which converge to $\mu_{0}$.

Lemma 2.4. The projection $E(t)$ converges to $E_{0}$ in norm.

Proof. This lemma follows from our earlier results that $E(t)$ converges strongly to $E_{0}$ and that for sufficiently small $t$, the dimension of $E(t)$ is exactly equal to that of $E_{0}$.

One of the most important tools for the identification of coefficients is the reduced resolvent.

Definition 2.1. Let $A$ be a self-adjoint operator with spectral measure $E(\lambda)$ and $\Gamma$ be an isolated spectral subset of $A$. Then the $\Gamma$-reduced resolvent of $A$ is defined as an operator valued function of $\rho$ for all $\rho$ in the union of $\Gamma$ and the resolvent set of $A$ by the equation $R_{\Gamma}(A, \rho)=\int^{\prime}(\lambda-\rho)^{-1} d E(\lambda)$ where the prime indicates that the integration is to extend over the complement of $\Gamma$.

Lemma 2.5. Let $\mu_{0}$ satisfy the conditions of Lemma 2.3 and let $\Gamma(t)$ be the set consisting of the $L$ eigenvalues of $\widetilde{H}(t)^{-1}$ which converge to $\mu_{0}$ for $t>0$ and $\Gamma(0)$ be the set with $\mu_{0}$ as its single element. For sufficiently small $t, S(t)=R_{\Gamma(t)}\left(\tilde{H}(t)^{-1}\right.$, $\mu_{0}$ ) defines an operator, and $S(t)$ is uniformly bounded, strongly convergent to $S(0)$, and satisfies the relation $S(t)\left\{\tilde{H}(t)^{-1}-\mu_{0}\right\}=I-E(t)$.

Proof. It is obvious that $S(t)$ is defined for small $t$. The cited properties 
follow from the operational calculus and the strong convergence of $\tilde{H}(t)^{-1}$ (proved in Theorem $3.1 \mathrm{~A}$ ).

We now list the inverse theorems from $A$ that we need for the present work. For any positive integer $i, A_{i}$ denotes the $i$ th term in the formal expansion of $H(t)^{-1}$; that is,

$$
A_{i}=\sum_{p_{1}+p_{2}+\cdots+p_{r}=i}(-1)^{r} H_{0}^{-1} H_{p_{1}} H_{0}^{-1} H_{p_{2}} H_{0}^{-1} \cdots H_{p_{r}} H_{0}^{-1} .
$$

Theorem 3.1 A. If $\phi \in \mathscr{D}\left(A_{i}\right)$ for $i \leq N$ and $A_{i} \phi \in D(H(t))$ for $i<N$, then $\lim _{t \rightarrow 0} t^{-N}\left\|\tilde{H}(t)^{-1} \phi-\sum_{i=0}^{N} t^{i} A_{i} \phi\right\|=0$.

Theorem $3.2 \mathrm{~A}$. If $\phi$ satisfies the conditions of Theorem $3.1 \mathrm{~A}$ and $A_{N} \phi \in D\left(\tilde{H}(t)^{1 / 2}\right)$, then $\lim _{t \rightarrow 0} t^{-(N+1 / 2)}\left\|\tilde{H}(t)^{-1} \phi-\sum_{i=0}^{N} t^{i} A_{i} \phi\right\|=0$.

TheOREM 4.1 A. If $\phi$ and $\psi$ satisfy the conditions of Theorem $3.1 \mathrm{~A}$ for order $N$ and $M$ respectively, then

$$
\begin{aligned}
\left(\tilde{H}(t)^{-1} \phi, \psi\right)= & \sum_{i=0}^{M-1} t^{i}\left(\phi, A_{i} \psi\right) \\
& -\sum_{j=M}^{M+N} t^{i} \sum_{k=M}^{j} \sum_{p=0}^{M-1}\left(A_{j-k} \phi, H_{k-p} A_{p} \psi\right) \\
& +o\left(t^{M+N}\right) .
\end{aligned}
$$

TheOREM 4.2 A. If $H(t)=H_{0}+t H_{1}$ and $A_{N} \phi$ and $A_{M} \psi$ are defined and belong to $D\left(\tilde{H}(t)^{1 / 2}\right)$, then

$$
\begin{aligned}
\left(\tilde{H}(t)^{-1} \phi, \psi\right)= & \sum_{i=0}^{M} t^{i}\left(\phi, A_{i} \psi\right) \\
& -\sum_{j=0}^{N-1} t^{M+j+1}\left(\left(H_{1} H_{0}^{-1}\right)^{j+1} \phi, A_{M} \psi\right)+t^{M+N+1}\left(\tilde{H}_{1}^{1 / 2} A_{N} \phi, \tilde{H}_{1}^{1 / 2} A_{M} \psi\right) \\
& +o\left(t^{M+N+1}\right) .
\end{aligned}
$$

We conclude this section by giving as a lemma the theorem which Kato uses as the basis for his investigation of asymptotic perturbation series. We also use the theorem but in an entirely different fashion.

LEMma 2.6. If $H$ is self-adjoint and $\lambda$ is an isolated nondegenerate eigenvalue and the interval $(\eta-d, \eta+d)$ contains no point other than $\lambda$ of the spectrum of $H$ and $\|(H-\eta) \omega\|=\epsilon$ and $\|\omega\|=1$ then $|\lambda-\eta| \leq \epsilon^{2} / d$ and there exists an eigenvector $\phi$ corresponding to $\lambda$ such that $\|\phi-\omega\| \leq \epsilon / d$. For fixed $\omega,\|(H-\eta) \omega\|$ assumes its minimum value for $\eta=(H \omega, \omega)$.

Proof. See Kato [1]. 
3. The nondegenerate case. For this section we assume $\mu_{0}$ satisfies the conditions of Lemma 2.3 with $L=1$ and $\phi_{0}$ is a corresponding normalized eigenvector. Then for sufficiently small $t, \widetilde{H}(t)^{-1}$ has an isolated eigenvalue $\mu(t)$ converging to $\mu_{0}$. The perturbed eigenvector is determined only to within a scalar factor. We obtain a unique eigenvector $\phi(t)$ by requiring $\phi(t)$ to be normalized and $\left(\phi(t), \phi_{0}\right)$ to be real and positive. The existence of $\phi(t)$ is clear since it is only a suitable scalar multiple of $E(t) \phi_{0}$. Note that $\phi(t)$ tends to $\phi_{0}$ as $t$ tends to zero. The following theorem gives a finite expansion for $\mu(t)$ and $\phi(t)$. We use $E_{0}$ and $S_{0}$ to denote $E(0)$ and $S(0)$ respectively.

THEOREM 3.1. If $\mu_{0}$ and $\phi_{0}$ are as above and $\mu_{1}=\left(A_{1} \phi_{0}, \phi_{0}\right), \phi_{1}=S_{0}\left(\mu_{1}-A_{1}\right) \phi_{0}$ and $\mu_{i}$ and $\phi_{i}$ are defined for $1<i \leq N$ by the recurrence relations

$$
\begin{aligned}
\mu_{i} & =\left(A_{i} \phi_{0}, \phi_{0}\right)-\sum_{j=1}^{i-1}\left(\left(\mu_{i-j}-A_{i-j}\right) \phi_{j}, \phi_{0}\right), \\
\left(I-E_{0}\right) \phi_{i} & =\sum_{j=0}^{i-1} S_{0}\left(\mu_{i-j}-A_{i-j}\right) \phi_{j}, \\
\left(\phi_{i}, \phi_{0}\right) & =-\frac{1}{2} \sum_{j=0}^{i-1}\left(\phi_{j}, \phi_{i-j}\right),
\end{aligned}
$$

and $A_{k} \phi_{i} \in \mathcal{D}(H(t))$ for $i+k<N$, then $\mu(t)=\mu_{0}+t \mu_{1}+\cdots+t^{N} \mu_{N}+o\left(t^{N}\right)$ and $\phi(t)=\phi_{0}+t \phi_{1}+\cdots+t^{N} \phi_{N}+o\left(t^{N}\right)$.

The proof is by induction. The case $N=0$ is contained in the previous discussion. Because the general proof is rather complicated we sketch a special proof for $N=1$ to help motivate the various steps. The perturbed eigenvalue and eigenvector can be expressed as $\mu(t)=\mu_{0}+\operatorname{tr}(t)$ and $\phi(t)=\phi_{0}+t \rho(t)$. We have to prove $\lim _{t \rightarrow 0} r(t)=\left(A_{1} \phi_{0}, \phi_{0}\right)$ and $\lim _{t \rightarrow 0} \rho(t)=S_{0}\left(\mu_{1}-A_{1}\right) \phi_{0}$.

From $\left[\widetilde{H}(t)^{-1}-\mu(t)\right] \phi(t)=0$ we obtain $\left[\widetilde{H}(t)^{-1}-\mu_{0}\right] \phi_{0}-\operatorname{tr}(t) \phi(t)+t\left[\widetilde{H}(t)^{-1}\right.$ $\left.-\mu_{0}\right] \rho(t)=0$. Since $\widetilde{H}(t)^{-1} \phi_{0}=A_{0} \phi_{0}+t A_{1} \phi_{0}+o(t)$ by Theorem $3.1 A$ and $A_{0} \phi_{0}$ $=H_{0}^{-1} \phi_{0}=\mu_{0} \phi_{0}$ this reduces to $r(t) \phi(t)=A_{1} \phi_{0}+\left[\widetilde{H}(t)^{-1}-\mu_{0}\right] \rho(t)+o(1)$. Now taking the inner product of both sides with $\phi_{0}$ and using the self-adjoint property of $\tilde{H}(t)^{-1}-\mu_{0}$ we obtain $r(t)\left(\phi(t), \phi_{0}\right)=\left(A_{1} \phi_{0}, \phi_{0}\right)+\left(\rho(t),\left[\widetilde{H}(t)^{-1}-\mu_{0}\right] \phi_{0}\right)$ $+o(1)$. The term involving $\left[\widetilde{H}(t)^{-1}-\mu_{0}\right] \phi_{0}$ can be reduced as above and the conclusion for $r(t)$ followed by taking limits as $t$ tends to zero.

Now we have $\mu(t)=\mu_{0}+t \mu_{1}+o(t)$ so the equation $\left[\tilde{H}(t)^{-1}-\mu(t)\right] \phi(t)=0$ can be reduced to $\left[\widetilde{H}(t)^{-1}-\mu(t)\right] \rho(t)=\left(\mu_{1}-A_{1}\right) \phi_{0}+o(t)$. Applying $S(t)$, the reduced resolvent of the perturbed operator, to both sides and taking limits we have $\lim _{t \rightarrow 0}[I-E(t)] \rho(t)=S_{0}\left(\mu_{1}-A_{1}\right) \phi_{0}$. Since $E(t)$ converges to $E_{0}{ }^{\prime}$ in norm, we can replace $E(t)$ by $E_{0}$ if we prove that $\rho(t)$ is bounded. This we accomplish by proving $\left(I-E_{0}\right) \rho(t)$ and $E_{0} \rho(t)$ bounded. From the equation $[I-E(t)] \phi(t)=0$ and the result that $[I-E(t)] \rho(t)$ is bounded we conclude $[I-E(t)] \phi_{0}=O(t)$. This together with $\phi_{0}=[I-E(t)] \phi_{0}+E(t) \phi_{0}$ yields 1 $=O\left(t^{2}\right)+\left|\left(\phi_{0}, \phi(t)\right)\right|^{2}$. Starting from $\phi(t)=\left(I-E_{0}\right) \phi(t)+E_{0} \phi(t)$ we obtain 
$1=\left\|\left(I-E_{0}\right) \phi(t)\right\|^{2}+\mid\left(\phi(t),\left.\phi_{0}\right|^{2}\right.$, and hence $\left\|\left(I-E_{0}\right) \phi(t)\right\|=O(t)$. This simplifies to yield $\left(I-E_{0}\right) \rho(t)$ bounded in the limit.

To prove $E_{0} \rho(t)$ bounded express $\phi(t)$ as $E_{0} \phi(t)+\left[I-E_{0}\right] \phi(t)$ and obtain the equation $1=\left\|E_{0} \phi(t)\right\|^{2}+\left\|\left[I-E_{0}\right] \phi(t)\right\|^{2}$. Since $\left[I-E_{0}\right] \phi(t)=O(t)$ we have $1-\left\|E_{0} \phi(t)\right\|^{2}=O\left(t^{2}\right)$ or $\left\{1-\left\|E_{0} \phi(t)\right\|\right\}\left\{1+\left\|E_{0} \phi(t)\right\|\right\}=O\left(t^{2}\right)$. Since the seconid factor tends to 2 we have $\left\|E_{0} \phi(t)\right\|=1+O\left(t^{2}\right)$ or $\left|\left(\phi(t), \phi_{0}\right)\right|=1+O\left(t^{2}\right)$. But $\phi(t)$ was determined by the condition that $\left(\phi(t), \phi_{0}\right)$ be positive so we have $\left(\phi(t), \phi_{0}\right)=1+O\left(t^{2}\right)$. From this it follows that $\left(\rho(t), \phi_{0}\right)=O(t)$ so $\lim _{t \rightarrow 0} E_{0} \rho(t)$ $=0$.

Thus $\rho(t)$ is bounded and $\lim _{t \rightarrow 0}\left(I-E_{0}\right) \rho(t)=S_{0}\left(\mu_{1}-A_{1}\right) \phi_{0}$ and $\lim _{t \rightarrow 0} E_{0} \rho(t)$ $=0$. This concludes the special proof for $N=1$.

Proof of Theorem 3.1. The theorem has been proved for $N=0,1$. We assume it true for $N-1$. Then we have $\mu(t)=\mu_{0}+t \mu_{1}+\cdots+t^{N-1} \mu_{N-1}+t^{N} r(t)$ and $\phi(t)=\phi_{0}+t \phi_{1}+\cdots+t^{N-1} \phi_{N-1}+t^{N} \rho(t)$. It remains to prove that $\lim _{t \rightarrow 0} r(t)=\mu_{N}$ and $\lim _{t \rightarrow 0} \rho(t)=\phi_{N}$ where $\mu_{N}$ and $\phi_{N}$ satisfy the relations (3.1), (3.2), and (3.3).

The remainder of the proof is divided into thirteen parts. The first three parts simply isolate the details of cancelling lower order terms from our main equations. The fourth part gives us the result for $r(t)$. In Part V we obtain an expression for $\lim _{t \rightarrow 0}[I-E(t)] \rho(t)$. As before for $N=1$ we can replace $E(t)$ by $E_{0}$ if we prove $\rho(t)$ bounded. The successful generalization of the device used before is to split $\rho(t)$ into its projections on the subspace determined by $\phi_{0}+t \phi_{1}+\cdots+t^{N-1} \phi_{N-1}$ and the complementary subspace. Parts VI through $\mathrm{X}$ are devoted to the proof that these are bounded. Part XI states the result for $\lim \left(I-E_{0}\right) \rho(t)$. In the twelf th part we calculate $\lim E_{0} \rho(t)$. The last part summarizes the results for $\rho(t)$.

I.

$$
\text { For } r<N, \quad \sum_{s=0}^{r}\left(A_{r-s}-\mu_{r-s}\right) \phi_{s}=0 .
$$

Proof. From (3.2) we obtain

$$
\left(A_{0}-\mu_{0}\right) \phi_{r}=\left(A_{0}-\mu_{0}\right) S_{0}\left[\sum_{s=0}^{r-1}\left(\mu_{r-s}-A_{r-s}\right) \phi_{s}\right]+\left(A_{0}-\mu_{0}\right) E_{0} \phi_{r} .
$$

Since $\left(A_{0}-\mu_{0}\right) S_{0}=I-E_{0}$ by Lemma 2.5 with $t=0$ and $\left(A_{0}-\mu_{0}\right) E_{0}$ $=\left(H_{0}^{-1}-\mu_{0}\right) E_{0}=0$, equation (3.4) reduces to

$$
\left(A_{0}-\mu_{0}\right) \phi_{r}=-\left(I-E_{0}\right)\left[\sum_{s=0}^{r-1}\left(A_{r-s}-\mu_{r-s}\right) \phi_{s}\right] .
$$

From (3.5) it follows that

$$
\sum_{s=0}^{r}\left(A_{r-s}-\mu_{r-s}\right) \phi_{s}=E_{0}\left[\sum_{s=0}^{r-1}\left(A_{r-s}-\mu_{r-s}\right) \phi_{s}\right] .
$$

By (3.1) we have 


$$
\begin{aligned}
\sum_{s=0}^{-1}\left(A_{r-.}-\mu_{r-s}\right) \phi_{s}= & A_{r} \phi_{0}-\mu_{r} \phi_{0}-\sum_{s=1}^{r-1}\left(A_{r-s}-\mu_{r-s}\right) \phi_{s} \\
= & A_{r} \phi_{0}-\left[\left(A_{r} \phi_{0}, \phi_{0}\right)-\sum_{s=1}^{r-1}\left(\left\{A_{r-s}-\mu_{r-s}\right\} \phi_{s}, \phi_{0}\right)\right] \phi_{0} \\
& -\sum_{s=1}^{r-1}\left(A_{r-s}-\mu_{r-s}\right) \phi_{s} .
\end{aligned}
$$

The inner product of $\phi_{0}$ with this last expression vanishes. Hence $E_{0}\left[\sum_{s=0}^{r-1}\left(A_{r-8}-\mu_{r-s}\right) \phi_{s}\right]=0$ and $I$ is established.

II. $\quad\left(\tilde{H}(t)^{-1}-\sum_{i=0}^{N} t^{i} \mu_{i}\right)\left(\sum_{j=0}^{N-1} t^{j} \phi_{j}\right)=t^{N} \sum_{k=0}^{N-1}\left(A_{N-k}-\mu_{N-k}\right) \phi_{k}+o\left(t^{N}\right)$.

Proof. By Theorem $3.1 A$ the formal expansion for $\tilde{H}(t)^{-1} \phi_{j}$ is valid to order $N-j$; that is $\widetilde{H}(t)^{-1} \phi_{j}=A_{0} \phi_{j}+t A_{1} \phi_{j}+\cdots+t^{N-i} A_{N-j} \phi_{j}+o\left(t^{N-j}\right)$. Substituting this expansion for $\widetilde{H}(t)^{-1} \phi_{j}$ for all $j$ and collecting terms we find that the coefficient of $t^{r}$ for $r<N$ is $\sum_{s=0}^{r}\left(A_{r-s}-\mu_{r-s}\right) \phi_{s}$ which vanishes by I.

III. $\quad\left(\tilde{H}(t)^{-1}-\sum_{i=0}^{N-1} t^{i} \mu_{i}\right)\left(\sum_{j=0}^{N-1} t^{j} \phi_{j}\right)=t^{N}\left\{\sum_{k=1}^{N-1}\left(A_{N-k}-\mu_{N-k}\right) \phi_{k}\right\}$

$$
+t^{N} A_{N} \phi_{0}+o\left(t^{N}\right) \text {. }
$$

Proof. Immediate from II. IV.

$$
\lim _{t \rightarrow 0} r(t)=\mu_{N}
$$

Proof. From $\left[\tilde{H}(t)^{-1}-\mu(t)\right] \phi(t)=0$ we obtain

$$
\left[\tilde{H}(t)^{-1}-\sum_{i=0}^{N-1} t^{i} \mu_{i}\right]\left(\sum_{j=0}^{N-1} t^{i} \phi_{j}\right)-t^{N} r(t) \phi(t)+t^{N}\left\{\tilde{H}(t)^{-1}-\sum_{i=0}^{N-1} t^{i} \mu_{i}\right\} \rho(t)=0
$$

which reduces to

$$
\begin{aligned}
\sum_{k=1}^{N-1}\left(A_{N-k}-\mu_{N-k}\right) \phi_{k}+A_{N} \phi_{0}+\left\{\tilde{H}(t)^{-1}-\sum_{i=0}^{N-1} t^{i} \mu_{i}\right\} \rho(t) \\
-r(t) \phi(t)+o(1)=0 .
\end{aligned}
$$

Taking the inner product with $\phi_{0}$ and using the self-adjoint property of $\widetilde{H}(t)^{-1}$ we obtain

$$
\begin{aligned}
r(t)\left(\phi(t), \phi_{0}\right)= & \left(A_{N} \phi_{0}, \phi_{0}\right)-\sum_{k=1}^{N-1}\left(\left(\mu_{N-k}-A_{N-k}\right) \phi_{k}, \phi_{0}\right) \\
& +\left(\rho(t),\left(H(t)^{-1}-\sum_{i=0}^{N-1} t^{i} \mu_{i}\right)=\phi_{0}\right)+o(1) .
\end{aligned}
$$


Since $\left(\tilde{H}(t)^{-1}-\sum_{i=0}^{N-1} t^{i} \mu_{i}\right) \phi_{0}=o(t)$ the term involving $\rho(t)$ tends to zero in the limit. By assumption $\lim _{t \rightarrow 0}\left(\phi(t), \phi_{0}\right)=1$ so the result follows on taking limits.

V. $\quad \lim _{t \rightarrow 0}\{I-E(t)\} \rho(t)=-\sum_{i=0}^{N-1} S_{0}\left(A_{N-i}-\mu_{N-i}\right) \phi_{i}$.

Proof. From $\left\{\widetilde{H}(t)^{-1}-\mu(t)\right\} \phi(t)=0$ and II we have

$$
\left\{\tilde{H}(t)^{-1}-\mu_{0}\right\} \rho(t)=-\sum_{i=0}^{N-1}\left(A_{N-i}-\mu_{N-i}\right) \phi_{i}+o(1) .
$$

Applying $S(t)$ to both sides of this equation we obtain

$$
\{I-E(t)\} \rho(t)=-\sum_{i=0}^{N-1} S(t)\left(A_{N-i}-\mu_{N-i}\right) \phi_{i}-S(t) o(1) .
$$

Part $\mathrm{V}$ follows from (3.7) and the uniform boundedness and strong convergence of $S(t)$.

Let $\sigma(t)=\phi_{0}+t \phi_{1}+\cdots+t^{N-1} \phi_{N-1}$ and $P(t)$ be the projection on the onedimensional subspace determined by $\sigma(t)$.

VI.

$$
\|\sigma(t)\|^{2}=1+t^{N} \sum_{i=1}^{N-1}\left(\phi_{i}, \phi_{N-i}\right)+o\left(t^{N}\right) .
$$

Proof. From (3.3) by direct calculation. VII.

$$
\|\{I-E(t)\} \sigma(t)\|=O\left(t^{N}\right) .
$$

Proof. This follows from the equation $\{I-E(t)\} \phi(t)=0$ and the boundedness of $\{I-E(t)\} \rho(t)$ from $\mathrm{V}$.

VIII.

$$
\|\{I-P(t)\} \phi(t)\|=O\left(t^{N}\right) .
$$

Proof. By direct calculation we obtain $\sigma(t)=\{I-E(t)\} \sigma(t)+(\sigma(t), \phi(t)) \phi(t)$ from which it follows that $1+O\left(t^{N}\right)=\|\{I-E(t)\} \sigma(t)\|^{2}+|(\sigma(t), \phi(t))|^{2}$ or $\|\{I-E(t)\} \sigma(t)\|^{2}=1-|(\sigma(t), \phi(t))|^{2}+O\left(t^{N}\right)$. Similarly, starting from the equation $\phi(t)=\{I-P(t)\} \phi(t)+P(t) \phi(t)$ we obtain $\|\{I-P(t)\} \phi(t)\|^{2}=1$ $-|(\sigma(t), \phi(t))|^{2}+O\left(t^{N}\right)$. Comparing this with the equation for $\|\{I-E(t) \phi(t) \|$ yields

$$
\|\{I-E(t)\} \sigma(t)\|^{2} .=\|\{I-P(t)\} \phi(t)\|^{2}+O\left(t^{N}\right) .
$$

Part VIII now follows from (3.9) and Part VII. IX.

$$
\|\{I-P(t)\} \rho(t)\|=O(1) .
$$

Proof. This is an immediate consequence of VIII and the relation $\{I-P(t)\} \rho(t)=-t^{-N}\{I-P(t)\} \phi(t)$.

$$
P(t) \rho(t)=O(1) .
$$


Proof. From $\phi(t)=P(t) \phi(t)+\{I-P(t)\} \phi(t)$ we obtain $1=\|P(t) \phi(t)\|^{2}$ $+\|\{I-P(t)\} \phi(t)\|^{2}$ so that $1-\|P(t) \phi(t)\|^{2}=O\left(t^{2 N}\right)$. Therefore $\{1-\|P(t) \phi(t)\|\}$ - $\{1+\|P(t) \phi(t)\|\}=O\left(t^{2 N}\right)$ and since the second factor tends to 2 it follows that $1-\|P(t) \phi(t)\|=O\left(t^{2 N}\right)$ or $\|\sigma(t)\|-2 \cdot|(\phi(t), \sigma(t))|=1+O\left(t^{2 N}\right)$. Expressing $\phi(t)$ as $\sigma(t)+t^{N} \rho(t)$ and using $\|\sigma(t)\|^{2}=1+O\left(t^{N}\right)$ from Part VI we obtain $(\rho(t), \sigma(t))$ bounded which is equivalent to $P(t) \rho(t)$ bounded.

XI.

$$
\lim _{t \rightarrow 0}\left(I-E_{0}\right) \rho(t)=\sum_{j=0}^{N-1} S_{0}\left(\mu_{N-j}-A_{N-j}\right) \phi_{j} .
$$

Proof. By IX and X, $\rho(t)$ is bounded. Obviously

$$
\left\|\left\{I-E_{0}\right\} \rho(t)-\{I-E(t)\} \rho(t)\right\| \leq\left\|E(t)-E_{0}\right\| \cdot\|\rho(t)\| .
$$

Part XI follows from Part V and the normed convergence of $E(t)$.

XII.

$$
\lim _{t \rightarrow 0} E_{0} \rho(t)=-\frac{1}{2} \sum_{j=1}^{N-1}\left(\phi_{j}, \phi_{N-i}\right) .
$$

Proof. We now have $\phi(t)=\sigma(t)+t^{N}\left\{I-E_{0}\right\} \phi_{N}+t^{N}\left(\rho(t), \phi_{0}\right) \phi_{0}+o\left(t^{N}\right)$ where $\rho$ is bounded. From the conditions that $\|\phi(t)\|=1$ and $\left(\phi(t), \phi_{0}\right)$ is real we conclude $\left(\rho(t), \phi_{0}\right)=2^{-1}\left\{1-\|\sigma(t)\|^{2}\right\}$. The desired result follows from Part VI.

XIII. $\quad \lim _{t \rightarrow 0} E_{0} \rho(t)=\phi_{N}$, where $\phi_{N}$ satisfies (3.2) and (3.3).

\section{Proof. From XI and XII.}

This concludes the proof of Theorem 3.1.

The complicated nature of the proof of the last theorem in comparison with the formal method is due to proving the existence of the eigenvector expansion. The first four parts are routine and follow the standard pattern. The formal counterpart of Part V would start from a formula for $\left(H_{0}^{-1}-\mu_{0}\right) \phi_{N}$, from which $\left(I-E_{0}\right) \phi_{N}$ is obtained by use of the unperturbed reduced resolvent. Our material is a formula for $\left\{\tilde{H}(t)^{-1}-\mu_{0}\right\} \rho(t)$, so we apply the perturbed reduced resolvent to obtain $\{I-E(t)\} \rho(t)$. To pass from this to $\left\{I-E_{0}\right\} \rho(t)$ we must prove that $\rho$ is bounded, and this causes most of the difficulty of the proof.

Also the formal method usually involves giving $E_{0} \phi_{N}$ an arbitrarily assigned value. We determine a unique eigenvector $\phi(t)$ by the condition that $\left(\phi(t), \phi_{0}\right)$ be real and then determine $E_{0} \phi_{N}$.

The hypotheses of Theorem 3.1 actually imply a much stronger conclusion with respect to the eigenvalues. To prove this we depart from the identification of coefficients and use Kato's theorem [Lemma 2.6].

THEOREM 3.2. Under the conditions of Theorem 3.1 the perturbed eigenvalue has an asymptotic expansion of order $2 N$.

Proof. Let $\omega(t)=\sum_{i=0}^{N} t^{i} \phi_{i}$ and $\eta(t)=\sum_{i=0}^{N} t^{i} \mu_{i}$. From Theorem 3.1 it fol- 
lows that $\left\{\tilde{H}(t)^{-1}-\eta(t)\right\} \omega(t)=o\left(t^{N}\right)$. Using Lemma 2.6 we conclude that the perturbed eigenvalue is equal to $\left(\widetilde{H}(t)^{-1} \omega(t), \omega(t)\right)$ to within terms of order $2 N$. This inner product is equal to

$$
\sum_{i=1}^{2 N} t^{i} \sum_{j=\max (0, i-N)}^{\min }\left(H(t)^{-1} \phi_{j}, \phi_{i-j}\right) .
$$

Since $\phi_{j}$ and $\phi_{i-j}$ satisfy the conditions of Theorem $4.1 A$ for orders $N-j$ and $N-(i-j)$ respectively, $\left(\widetilde{H}(t)^{-1} \phi_{j}, \phi_{i-j}\right)$ can be expanded to order $2 N-i$. We would obtain the desired expansion by expanding each of these inner products and collecting terms.

We obtain an odd order expansion of the eigenvalue by combining a modification of the identification of coefficients with Theorems $3.2 \mathrm{~A}$ and 4.2 A. For simplicity the result is stated for operators of the form $H_{0}+t H_{1}$ but it can be extended easily.

ThEOREM 3.3. If $H(t)=H_{0}+t H_{1}$ and the conditions of Theorem 3.1 are satisfied and in addition $A_{k} \phi_{i} \in D\left(\widetilde{H}(t)^{1 / 2}\right)$ for $i+k=N$, then the perturbed eigenvalue has an asymptotic expansion of order $2 N+1$.

Proof. Let $\omega(t)=\sum_{i=0}^{N} t^{i} \phi_{i}$ and $\nu(t)$ be the expansion of $\mu(t)$ to order $2 N$ obtained in Theorem 3.2. By Theorem 3.2A we have $\left\|\left\{\tilde{H}(t)^{-1}-\nu(t)\right\} \omega(t)\right\|$ $=o\left(t^{N+1 / 2}\right)$. By Lemma 2.6, this implies that $\mu(t)=\left(\tilde{H}(t)^{-1} \omega(t), \omega(t)\right)$ to within terms of order $2 N+1$. Using Theorem $4.2 A$ we can expand this inner product to order $2 N+1$.

Example 3.1. An interesting example in connection with Theorem 3.1 is furnished by the operator associated with the vibrating rod of small stiffness clamped at both ends, $H(\epsilon) \phi=d^{2} / d x^{2}+\epsilon\left(d^{4} / d x^{2}\right)$ for $\epsilon>0$ with boundary conditions $\phi(0)=\phi(1)=\phi^{\prime}(0)=\phi^{\prime}(1)=0$. For convenience we discuss the operator itself instead of its inverse. The Friedrichs extension of the second derivative subject to the above boundary conditions is obtained by relaxing the conditions to $\phi(0)=\phi(1)=0$. Therefore we select the second derivative with these boundary conditions as $H_{0}$. Then the smallest eigenvalue of $H_{0}$ is $\lambda_{0}=\pi^{2}$ with corresponding eigenvector $\phi_{0}=2^{1 / 2} \sin \pi x$.

We can consider the perturbed operator as being of the form $H_{0}+t H_{1}$ with $H_{1}$ the fourth derivative with boundary conditions $\phi(0)=\phi(1)=\phi^{\prime}(0)$ $=\phi^{\prime}(1)=0$. In this case $\left(H_{1} \phi_{0}, \phi_{0}\right)$, the first term of the formal expansion for $\lambda(t)$, is not even defined since $\phi_{0}$ does not satisfy the boundary conditions for $H_{1}$.

We can also consider the perturbed operator as being of the form $H_{0}+t H_{1}$ $+t^{2} H_{2}$, with $H_{1}$ the zero operator and $H_{2}$ the fourth derivative. In this case the first term of the formal expansion is defined with value zero. But the expansion $\lambda(t)=\lambda_{0}+0 \cdot t+o(t)$ is not valid; the true expansion is

$$
\lambda(t)=\lambda_{0}+4 \pi^{2} / t+o(t) .
$$


(Rayleigh [1, p. 300]). The conditions of Theorem 3.1 for $N=1$ are not satisfied since $A_{0} \phi_{0}$ is not in the domain of $H(t)$.

If we specialize the theorems of this section to the case $H(t)=H_{0}+t H_{1}$ we obtain Kato's results for the corresponding case. Kato also studied essentially self-adjoint perturbed operators. Such operators might not have inverses so the methods of the present paper cannot be applied.

4. First order splitting; eigenvalues. For this section we assume $\mu_{0}$ satisfies the conditions of Lemma 2.3 with $L>1$. We denote orthonormal unperturbed eigenvectors by $\phi_{0}^{1}, \cdots, \phi_{0}^{L}$. For sufficiently small $t, \tilde{H}(t)^{-1}$ has $L$ eigenvalues converging to $\mu_{0}$ which we order according to magnitude; $\mu^{1}(t) \leq \mu^{2}(t) \leq \ldots$. $\leq \mu^{L}(t)$. The following theorem gives a first order expansion of the eigenvalues and a zero order result for eigenvectors. Further expansion of the eigenvectors will be considered in the next section.

THEOREM 4.1. If $\phi_{0}^{k} \in \mathcal{D}(H(t))$ for all $k$, then we may assume $\phi_{0}^{k}$ selected so that $\left(A_{1} \phi_{0}^{i}, \phi_{0}^{j}\right)=\delta_{i j} \mu_{1}^{j}$ with $\mu_{1}^{1} \leq \mu_{1}^{2} \leq \cdots \leq \mu_{1}^{L}$. The perturbed eigenvalues satisfy $\mu^{j}(t)=\mu_{0}+t \mu_{1}^{j}+o(t)$. If the $\mu_{1}^{j}$ are distinct, then there exist perturbed eigenvectors $\phi^{i}(t)$ such that $\lim _{t \rightarrow 0} \phi^{j}(t)=\phi_{0}^{j}$ for all $j$.

Proof. Since $\lim _{t \rightarrow 0} \mu^{i}(t)=\mu_{0}$, we can express $\mu^{j}(t)$ as $\mu^{i}(t)=\mu_{0}+t r^{i}(t)$. We must prove $\lim _{t \rightarrow 0} r^{i}(t)=\mu_{1}^{j}$. Since $E(t)$ converges to $E_{0}$, there exist perturbed eigenvectors of the form

$$
\psi^{i}(t)=\sum_{i=1}^{L} c_{j i}(t) \phi_{0}^{i}+t_{\rho}^{j}(t)
$$

where $\rho^{j}(t)$ is orthogonal to all $\phi_{0}^{k}$ and $\lim _{t \rightarrow 0} t \rho^{i}(t)=0$. By taking the norm of both sides of (4.1) we conclude that each $c_{j i}(t)$ is bounded and that $\sum_{i=1}^{L}\left|c_{j i}(t)\right|^{2}$ is bounded away from zero in the limit.

Obviously,

$$
\left(\psi^{i}(t),\left\{\widetilde{H}(t)^{-1}-\mu^{j}(t)\right\} \phi_{0}^{k}\right)=\left(\left\{\widetilde{H}(t)^{-1}-\mu^{j}(t)\right\} \psi^{j}(t), \phi_{0}^{k}\right)=0 .
$$

Expanding $\widetilde{H}(t)^{-1} \phi_{0}^{k}$ to first order and simplifying, we obtain

$$
\left\{\begin{array}{l}
k \\
\mu_{1}
\end{array}-r^{j}(t)\right\} c_{j k}(t)+\sum_{i=1}^{L} c_{j i}(t) o_{i j k}(1)=-\left(t_{\rho}^{j}(t), A_{1} \phi_{0}^{k}+o_{j k}(1)\right) .
$$

Note that the right hand side of (4.2) vanishes in the limit.

From (4.2) we conclude that the $r^{i}(t)$ are bounded in the limit. Suppose to the contrary that some $r^{u}(t)$ becomes unbounded on a sequence $\left\{t_{n}\right\}$ of parameter values tending to zero. Since $\lim _{t \rightarrow 0} \sum_{k=1}^{L}\left|c_{\boldsymbol{u} k}(t)\right|^{2}=1$, there exists a $v$ and a subsequence $\left\{t_{n}^{\prime}\right\}$ such that $c_{u v}\left(t_{n}^{\prime}\right)$ remains bounded away from zero. Then the first term in (4.2) for $j=u, k=v$ will become unbounded as $t$ takes on the values $t_{n}^{\prime}$. On the other hand, all remaining terms are bounded, 
so the first must be also. Thus the assumption that $r^{u}(t)$ becomes unbounded leads to a contradiction.

Now regard (4.2) as a system of $L$ nonhomogeneous equations in the $L$ unknowns $c_{j k}$ ( $j$ fixed, $k$ varying). For any $t$ such that the determinant of coefficients does not vanish, each $c_{j k}(t)$ must be the quotient of two determinants. Each numerator determinant will have one column of terms which tend to zero and other terms bounded. Therefore, each numerator tends to zero. Since $\sum_{i=1}^{L}\left|c_{j i}(t)\right|^{2}$ is bounded away from zero in the limit, the denominator must tend to zero. This results in the condition

$$
\lim _{t \rightarrow 0} \prod_{k=1}^{L}\left\{\mu_{1}^{k}-r^{j}(t)\right\}=0 \text { for each } j .
$$

Let $C$ be a constant which is not a characteristic root of the matrix $\left(A_{1} \phi_{0}^{i}, \phi_{0}^{j}\right)$ and let $\left\{t_{n}\right\}$ be a sequence of parameter values tending to zero such that for each $j, r^{i}\left(t_{n}\right)$ is either less than $C$ or greater than $C$ for all $n$. From (4.2) it follows that if $r^{j}\left(t_{n}\right)-\mu_{1}^{k}$ is bounded away from zero, then $\lim _{n \rightarrow \infty} c_{j k}\left(t_{n}\right)$ $=0$. From this we see that the $\phi^{i}\left(t_{n}\right)$ corresponding to those $r^{j}\left(t_{n}\right)$ which are less than $C$ [greater than $C$ ] determine a projection which converges strongly to that determined by those $\phi_{0}^{j}$ such that $\left(A_{1} \phi_{0}^{j}, \phi_{0}^{j}\right)$ is less than $C$ [greater than $C$ ]. It follows that the number of distinct $j$ 's such that $r^{j}\left(t_{n}\right)$ is less than $C$ [greater than $C$ ] is not less than the sum of the multiplicities of the characteristic roots which are less than $C$ [greater than $C$ ]. Since the total number of $r$ 's is equal to the total multiplicity of all roots, the equality must hold. From this result and (4.3) it follows that $\lim _{t \rightarrow 0} r^{j}(t)=\mu_{1}^{j}$ for all $j$.

The second conclusion of the theorem follows immediately from (4.2).

REMARK. If $A_{1} \phi_{0}^{i}$ is defined, then $\widetilde{H}_{1}^{1 / 2} \phi_{0}^{i}$ is also, but the converse is not true. If we assume $\phi_{0}^{j} \in \mathscr{D}\left(\widetilde{H}(t)^{1 / 2}\right)$ for all $j$, we can prove that the first order terms in the expansions of the eigenvalues are the characteristic roots of the matrix with $-\left(\widetilde{H}_{1}^{1 / 2} H_{0}^{-1} \phi_{0}^{i}, \widetilde{H}_{1}^{1 / 2} H_{0}^{-1} \phi_{0}^{j}\right)=-\left(\mu_{0}\right)^{2}\left(\widetilde{H}_{1}^{1 / 2} \phi_{0}^{i}, \widetilde{H}_{1}^{1 / 2} \phi_{0}^{j}\right)$ in the $i, j$ th position. We do not obtain conclusions with respect to eigenvectors in this case. The proof depends on a generalization of Lemma 2.6 and will be omitted.

5. First order splitting; eigenvectors. We now proceed to the study of the identification of coefficients as applied to the calculation of eigenvectors in the degenerate case. The first order result of the formal method is that if the numbers $\mu_{1}^{j}$ of Theorem 4.1 are distinct, then the perturbed eigenvectors are of the form $\phi^{i}(t)=\phi_{0}^{j}+t \phi_{1}^{j} \cdots$ with $\left(I-E_{0}\right) \phi_{1}^{j}=-S_{0} A_{1} \phi_{0}^{j}$. With first order inverse assumptions there exist perturbed eigenvectors $\phi^{j}(t)=\phi_{0}^{j}+t \omega^{j}(t)$ (Theorem 4.1). To obtain unique vectors we impose the condition that $\left(\phi^{i}(t), \phi_{0}^{j}\right)$ be real. With no additional assumptions we easily prove $\{I-E(t)\} \omega^{j}(t)$ $=-S_{0} A_{1} \phi_{0}^{j}+o(t)$ (Lemma 5.1). In order to replace $E(t)$ by $E_{0}$ we must prove $\omega^{j}(t)$ bounded. Our success in the nondegenerate case suggests that we attempt to prove $\left(I-E_{0}\right) \omega^{j}(t)$ and $E_{0} \omega^{j}(t)$ bounded separately. The first of these causes no difficulty (Lemma 5.2), but the author is unable to prove the 
second bounded with first order assumptions only. (Note that in the formal method the corresponding term is computed at second order.) With second order assumptions it is not difficult to prove $E_{0} \omega^{j}(t)$ bounded, (Lemmas 5.3 and 5.4), but we do not obtain its limiting value at this point. Now we have $\omega^{j}(t)$ bounded, so we conclude $\left\{I-E_{0}\right\} \omega^{j}(t)=-S_{0} A_{1} \phi_{0}^{j}+o(t)$ (Lemma 5.5). This result in turn makes it possible to verify the formal result for $\lim _{t \rightarrow 0} E_{0} \omega^{j}(t)$ (Lemma 5.6). We also verify the formal result for the second order term for the eigenvalue (Lemma 5.7). All results are collected in Theorem 5.1.

Lemma 5.1. With the hypotheses of Theorem 4.1;

$$
\{I-E(t)\} \omega^{j}(t)=-S_{0} A_{1} \phi_{0}^{j}+o(t) .
$$

Proof. By expanding the equation $\left\{\widetilde{H}(t)^{-1}-\mu^{j}(t)\right\} \phi^{j}(t)=0$ and cancelling lower order terms we obtain $\left\{\widetilde{H}(t)^{-1}-\mu_{0}\right\} \omega^{j}(t)=-A_{1} \phi_{0}^{j}+\mu_{1}^{j} \phi_{0}^{j}+o(1)$. Applying the reduced resolvent $S(t)$ to both sides of this equation and taking the limit with respect to $t$ we obtain the desired result.

Lemma 5.2. With the hypotheses of Theorem 4.1, $\left\|\left(I-E_{0}\right) \omega^{j}(t)\right\|=O(1)$.

Proof. From Lemma 5.1 and the equation $\{I-E(t)\} \phi^{i}(t)=0$, we obtain $\left\|\{I-E(t)\} \phi_{0}^{y}\right\|=O(t)$. By direct calculation we have

$$
\begin{aligned}
& \left\|\left(I-E_{0}\right) \phi^{i}(t)\right\|^{2}=1-\sum_{i=1}^{L}\left|\left(\phi^{i}(t), \phi_{0}^{i}\right)\right|^{2}, \\
& \left\|(I-E(t)) \phi_{0}^{j}\right\|^{2}=1-\sum_{i=1}^{L}\left|\left(\phi_{0}^{j}, \phi^{i}(t)\right)\right|^{2} .
\end{aligned}
$$

Summing the last two equations yields

$$
\sum_{j=1}^{L}\left\|\left(I-E_{0}\right) \phi^{i}(t)\right\|^{2}=L-\sum_{i, j=1}^{L}\left|\left(\phi^{j}(t), \phi_{0}^{i}\right)\right|^{2}=\sum_{j=1}^{L}\left\|(I-E(t)) \phi_{0}^{i}\right\|^{2}
$$

so that $\left\|\left(I-E_{0}\right) \phi^{j}(t)\right\|=O(t)$, which is equivalent to the statement of the lemma.

Lemma 5.3. If the conditions of Theorem 4.1 hold and in addition $A_{1} \phi_{0}^{j}$ $\in \mathbb{D}(H(t))$ for all $j$, then $\left(\omega^{j}(t), \phi_{0}^{k}\right)=O(t)$ for $j \neq k$.

Proof. Note that $\left(\omega^{j}(t), \phi_{0}^{k}\right)$ is equal in absolute value to the $c_{j k}(t)$ of Theorem 4.1. Because of the additional assumptions on $\phi_{0}^{j}$, we can replace the terms $o_{i j k}(1)$ and $o_{j k}(1)$ in $(4.2)$ by $O_{i j k}(t)$ and $O_{j k}(t)$,

$$
\left\{\begin{array}{c}
k \\
\mu_{1}
\end{array}-r^{j}(t)\right\} c_{j k}(t)+\sum_{i=1}^{L} c_{j i}(t) O_{i j k}(t)=-\left(t_{\rho}^{i}(t), A_{1} \phi_{0}^{k}-O_{j k}(t)\right) .
$$

From $\left(A_{1} \phi_{0}^{i}, \phi_{0}^{j}\right)=\delta_{i j} \mu_{1}^{1}$ it follows that 


$$
\left(t_{\rho}^{i}(t), A_{1} \phi_{0}^{k}\right)=\left(\psi^{j}(t),\left(A_{1}-\mu_{1}^{k}\right) \phi_{0}^{k}\right)\left(\left\{I-E_{0}\right\} \phi^{j}(t),\left\{A_{1}-\mu_{1}^{k}\right\} \phi_{0}^{k}\right) .
$$

From (5.1) and (5.2) we obtain

$$
\begin{aligned}
\sum_{i=1}^{L} t^{-1} c_{j i}(t)\left\{\delta_{i k}\left(\mu_{1}^{k}-r_{1}^{j}(t)\right)+O_{i j k}(t)\right\} & \\
= & -\left(t^{-1}\left\{I-E_{0}\right\} \phi^{j}(t),\left\{A_{1}-\mu_{1}^{k}\right\} \phi_{0}^{k}\right)-\left(t \rho^{j}(t), O(1)\right) .
\end{aligned}
$$

Regard (5.3) as $L-1$ equations in $L-1$ unknowns $t^{-1} c_{j k}(t), j$ fixed, $j \neq k$. The coefficients are bounded in the limit and the determinant is bounded away from zero in the limit. Therefore, $t^{-1} c_{j k}(t)$ is bounded in the limit for $j \neq k$.

Lemma 5.4. With the hypotheses of Lemma $5.3, \lim _{t \rightarrow 0}\left(\omega^{i}(t), \phi_{0}^{j}\right)=0$.

Proof. Expanding $\phi^{j}(t)=E_{0} \phi^{j}(t)-\left(I-E_{0}\right) \phi^{j}(t)$ we obtain

$$
\phi^{j}(t)=\left(\phi^{j}(t), \phi_{0}^{j}\right)+\sum_{k=1, k \neq i}^{L} c_{j k}(t) \phi_{0}^{k}-\left(I-E_{0}\right) \phi^{j}(t) .
$$

Calculating the norm of each side yields

$$
1=\left|\left(\phi^{j}(t), \phi_{0}^{j}\right)\right|^{2}+\sum_{k=1, k \neq j}^{L}\left|c_{j k}(t)\right|^{2}+\left\|\left(I-E_{0}\right) \phi^{j}(t)\right\|^{2} .
$$

Applying the two preceding lemmas we obtain $1-\left|\left(\phi^{j}(t), \phi_{0}^{j}\right)\right|^{2}=O\left(t^{2}\right)$. Since $\left(\phi^{i}(t), \phi_{0}^{j}\right)$ is real and tends to one in the limit, $1-\left|\left(\phi^{j}(t), \phi_{0}^{j}\right)\right|^{2}=O\left(t^{2}\right)$. The statement of the lemma follows from this last equation.

Lemma 5.5. With the hypotheses of Lemma 5.3,

$$
\left(I-E_{0}\right) \omega^{i}(t)=-S_{0} A_{1} \phi_{0}^{i}+o(1) .
$$

Proof. By the three preceding lemmas, $\omega^{i}(t)$ is bounded. The desired result follows from Lemma 5.1 and the normed convergence of $E(t)$.

Lemma 5.6. With the hypotheses of Lemma 5.3,

$$
\lim _{t \rightarrow 0}\left(\omega^{i}(t), \phi_{0}^{k}\right)=\left(\mu_{1}^{k}-\mu_{1}^{j}\right)^{-1}\left\{\left(S_{0} A_{1} \phi_{0}^{j}, A_{1} \phi_{0}^{k}\right)-\left(\phi_{0}^{i}, A_{2} \phi_{0}^{k}\right)\right\}
$$

for $j \neq k$.

Proof. Expressing $\mu^{i}(t)$ in the form $\mu_{0}+t \mu_{1}^{j}+t^{2} r^{i}(t)$ and expanding the equation $\left(\phi^{j}(t),\left\{\widetilde{H}(t)^{-1}-\mu^{j}(t)\right\} \phi_{0}^{k}\right)=0$, we obtain

$$
\begin{aligned}
& \left(\phi_{0}^{j}-t S_{0} A_{1} \phi_{0}^{j}+t \sum_{i=1}^{L}\left(\omega^{j}, \phi_{0}^{i}\right) \phi_{0}^{i}+o(t),\right. \\
& \left.t A_{1} \phi_{0}^{k}+t^{2} A_{2} \phi_{0}^{k}-t \mu_{1}^{j} \phi_{0}^{k}-t^{2} r^{i}(t)\right)=0 .
\end{aligned}
$$


For $j \neq k$, this reduces to

$$
\left(\omega^{j}(t), \phi_{0}^{k}\right)\left\{{ }_{\mu_{1}}^{k}-\mu_{1}^{j}\right\}+o(t)+\left(\phi_{0}^{j}, A_{2} \phi_{0}^{k}\right)-\left(S_{0} A_{1} \phi_{0}^{j}, A_{1} \phi_{0}^{k}\right)=0 .
$$

Taking the limit with respect to $t$ we obtain the conclusion of the lemma.

Lemma 5.7. With the hypotheses of Lemma 5.3,

$$
\mu^{j}(t)=\mu_{0}+t \mu_{1}^{j}+t^{2} \mu_{2}^{j}+o\left(t^{2}\right)
$$

with

$$
\stackrel{j}{\mu_{2}}=-\left(S_{0} A_{1} \phi_{0}^{j}, A_{1} \phi_{0}^{j}\right)-\left(\phi_{0}^{i}, A_{2} \phi_{0}^{j}\right) .
$$

Proof. Immediate from (5.4) for $j=k$.

The preceding results are collected to yield the following theorem.

TheOREM 5.1. If $\phi_{0}^{j}, A_{1} \phi_{0}^{j} \in \mathfrak{D}(H(t))$ for all $j$, then the perturbed eigenvalues $\mu^{i}(t)$ and eigenvectors $\phi^{j}(t)$ determined as above satisfy

$$
\begin{aligned}
& \mu^{j}(t)=\mu_{0}+t \mu_{1}^{j}+t^{2} \mu_{2}^{j}+o\left(t^{2}\right), \\
& \phi^{j}(t)=\phi_{0}^{j}+t \phi_{1}^{j}+o(t)
\end{aligned}
$$

with

$$
\begin{aligned}
\mu_{1}^{j} & =\left(A_{1} \phi_{0}^{j}, \phi_{0}^{j}\right), \\
\left(I-E_{0}\right) \phi_{1}^{j} & =-S A_{1} \phi_{0}^{j}, \\
\left(\phi_{1}^{j}, \phi_{0}^{k}\right) & =\left(\mu_{1}^{k}-\mu_{1}^{j}\right)^{-1}\left\{\left(S_{0} A_{1} \phi_{0}^{j}, A_{1} \phi_{0}^{k}\right)-\left(\phi_{0}^{j}, A_{2} \phi_{0}^{k}\right)\right\}, \\
\mu_{2}^{j} & =-\left(S_{0} A_{1} \phi_{0}^{j}, A_{1} \phi_{0}^{j}\right)+\left(\phi_{0}^{j}, A_{2} \phi_{0}^{j}\right) .
\end{aligned}
$$

A completely general discussion of splitting at any order would be extremely tedious. We consider only the simplest case, that in which the eigenvalue either does not split at all or else splits completely at any order. At any order, whether the eigenvalue splits or not is determined by comparing the characteristic roots of a matrix peculiar to that order. As the order increases, the associated matrix becomes more and more complicated, but it always remains symmetric. If we made the indlictive assumption that the matrices encountered at orders less than $N$ have equal characteristic roots they would have to be multiples of the identity. This simple observation would allow us to cancel all terms of degree less than $N$ from the equation

$$
\left(\left\{\tilde{H}(t)^{-1}-\mu^{j}(t)\right\} \phi^{j}(t), \phi_{0}^{k}\right)=0
$$

and so pass on to the proof that the $N$ th terms in the expansion of the eigenvalues are determined by the characteristic roots of the $N$ th order matrix. 
Even for operators of the form $H_{0}+t H_{1}$, an eigenvalue can split at second order. An example is

$$
H(t)=\left(\begin{array}{lll}
1 & 0 & 0 \\
0 & 1 & 0 \\
0 & 0 & 2
\end{array}\right)+t\left(\begin{array}{rrr}
0 & 0 & i \\
0 & 0 & 0 \\
-i & 0 & 0
\end{array}\right) .
$$

An extreme example of splitting is given by the matrix

$$
H(t)=\left(\begin{array}{rr}
1-f \cos g & -f \sin g \\
-f \sin g & 1+f \cos g
\end{array}\right)
$$

with $f=\exp \left(-t^{-2}\right)$ and $g=2 / t$. This matrix does not have a convergent series expansion in powers of $t$ but the expansion $H(t)=I+0 \cdot t+0 \cdot t^{2}+\cdots$ is valid asymptotically. For $t>0$, the matrix has two distinct eigenvalues $1-f$ and $1+f$ with the same asymptotic expansion. The eigenvalue splits but the splitting does not occur at a finite order. Another interesting property of this matrix is that it is impossible to select perturbed eigenvectors which converge as $t$ tends to zero.

6. The adiabatic transformation. Let $H_{0}$ be a self-adjoint operator, $\lambda_{0}$ an isolated eigenvalue of $H_{0}$ of multiplicity $L$, and $E_{0}$ the projection on the corresponding eigenspace. Let $H(t)$ be a self-adjoint perturbation of $H_{0}$ such that for $t$ sufficiently small, the spectrum of $H(t)$ near $\lambda_{0}$ consists of one eigenvalue of multiplicity $L$. (That is, $\lambda_{0}$ does not split under the perturbation.) Denote the projection on the corresponding eigenspace by $E(t)$.

Perturbed eigenvectors are not uniquely determined by the conditions that they be orthonormal and converge to previously selected unperturbed eigenvectors. In fact, the quantities $\left(\phi_{n}^{j}, \phi_{0}^{i}\right)$ are determined only to within real part. We find it convenient to obtain unique eigenvectors by insisting such terms be real, but this is an arbitrary and somewhat artificial requirement. In this section we introduce the notion of the adiabatic transformation which gives rise to naturally distinguished eigenvectors.

Kato [4] has shown that if $E(t)$ is of class $C^{2}$ as a function of $t$, then there exists a unitary operator $U(t)$ defined on all of $\mathfrak{F}$ which maps the range of $E_{0}$ onto the range of $E(t)$. This operator is called the adiabatic transformation corresponding to $\lambda(t)$ and the correspondence from selected $\phi_{0}^{j}$ to the vectors $\phi^{i}(t)=U(t) \phi_{0}^{j}$ is called the adiabatic change of eigenvectors. Note that we can still select the $\phi_{0}^{j}$ arbitrarily. It is only the change in eigenvectors that is distinguished.

The operator $U$ is defined as the solution of the differential equation

$$
U^{\prime}=\left(E^{\prime} E-E E^{\prime}\right) U
$$

which takes on the initial value $I$ for $t=0$. From the existence of $L$ orthonormal perturbed eigenvectors with $N$ th $(N \geq 2)$ order asymptotic expansions 
follows the existence of eigenvectors in the adiabatic form with the same property.

If we define $W(t)$ as $U(t) E_{0}$, then $W \phi$ will be equal to $U \phi$ for all $\phi$ in the range of $E_{0}$, and $W$ satisfies the simpler differential equation $W^{\prime}=E^{\prime} W$ (see Kato [4]). In particular

$$
W^{\prime} \phi_{0}^{j}=E^{\prime} \phi^{j}(t) .
$$

THEOREM 6.1. Let the perturbed eigenvectors in adiabatic form have the expansion

$$
\phi^{i}(t)=\phi_{0}^{i}+t \phi_{1}^{i}+t^{2} \phi_{2}^{j}+\cdots+t^{N} \phi_{N}^{i}+o\left(t^{N}\right) .
$$

Then

$$
\left(\phi_{k}^{j}, \phi_{0}^{i}\right)=-1 / k \sum_{r=1}^{k-1} r\left(\phi_{r}^{j}, \phi_{k-r}^{i}\right)
$$

Proof. Using the given expansion to evaluate both sides of (6.1) and then taking the $(k-1)$ st derivative at $t=0$, we obtain

$$
\sum_{j=1}^{L}\left\{\sum_{r=1}^{k}\left(\phi_{0}^{i}, \phi_{r}^{j}\right) \phi_{k-r}^{j}+1 / k \sum_{s=1}^{k-1} s \sum_{u=0}^{s}\left(\phi_{k-s}^{i}, \phi_{u}^{j}\right) \phi_{s-u}^{j}\right\}=0 .
$$

For $k=1$, the theorem follows immediately from the linear independence of the vectors $\phi_{0}^{j}$. Assuming it holds for $k<n$ we can prove that the coefficient of $\phi_{s}^{j}$ in (6.2) vanishes for $s \geq 1$. Therefore, the coefficient of $\phi_{0}^{j}$ must also vanish which yields the theorem for $k=n$, completing the induction.

In the degenerate case, we use this formula only when splitting does not occur. The result for $\left(\phi_{0}^{i}, \phi_{1}^{j}\right)$ in this section does not agree with that in the previous section where the eigenvalue did split at first order. In the nondegenerate case we obtain the perturbed eigenvector in adiabatic form by replacing (3.3) by

$$
\left(\phi_{i}, \phi_{0}\right)=-1 / i \sum_{j=1}^{i-1} j\left(\phi_{j}, \phi_{i-j}\right) .
$$

\section{BIBLIOGRAPHY}

K. Friedrichs

1. Spéktraltheorie halbbeschränkter Operatoren und Anwendung auf die Spektralzerlegung von Differentialoperatoren, Math. Ann. vol. 109 (1934) pp. 465-487.

2. Spektraltheorie halbbeschränkter Operatoren, II, Math. Ann. vol. 109 (1934) pp. 685713.

T. Kato

1. On the upper and lower bounds of eigenvalues, Journal of the Physical Society of Japan vol. 4 (1949) pp. 334-339.

2. On the convergence of the perturbation method, J. Fac. Sci. Imp. Univ. Tokyo. Sect. I vol. VI, part 3, pp. 145-226. 
3. Perturbation theory of semi-bounded operators, Math. Ann. vol. 125 (1953) pp. 435-447.

4. On the adiabatic theorem of quantum mechanics, Journal of the Physical Society of Japan V. Kramer vol. 5 (1950) pp. 435-439.

1. Asymptotic inverse series, Proc. Amer. Math. Soc. vol. 7 (1956) pp. 429-437.

B. de Sz.-Nagy

1. Perturbations des transformations autoadjointes dans l'espace de Hilbert, Comment. Math. Helv. vol. 19 (1947) pp. 347-366.

L. C. Pauling and E. B. Wilson

1. Introduction to quantum mechanics, New York, 1935.

Lord Rayleigh

1. Theory of sound I, 2d ed. rev., London, 1927, Macmillan.

F. Rellich

1. Störungstheorie der Spektralzerlegung I., Math. Ann. vol. 113 (1937) pp. 600-619.

2. II. Math. Ann. vol. 113 (1937) pp. 677-685.

3. III. Math. Ann. vol. 116 (1939) pp. 555-570.

4. IV. Math. Ann. vol. 117 (1940) pp. 356-382.

5. Halbbeschränkte gewohnliche Differentialoperatoren zweiter Ordnung, Math. Ann. vol. 122 (1951) pp. 343-368.

M. Stone

1. Linear transformations in Hilbert space, Amer. Math. Soc. Colloquium Publications, vol. 15, New York, 1932.

E. C. Titchmarsh

1. Some theorems on perturbation theory, Proc. Roy. Soc. London Ser. A vol. 200 (1949) pp. 24-46.

F. Wolf

1. Analytic perturbation of operators in Banach spaces, Math. Ann. vol. 124 (1951) pp. 317333.

University of California, Berkeley, Calif. 